\title{
Co-diseño de una aplicación para el reconocimiento in situ del gorgojo de los andes en cultivos de papa
}

Resumen-Diversos métodos son empleados evitar que los cultivos de papa se vean afectados por enfermedades y plagas, uno de estos, es el monitoreo que consiste en personas encargadas de recorrer los cultivos y emplear sus capacidades cognitivas para reconocer la presencia de plagas. Sin embargo, limitaciones en la capacidad humana como la imprecisión por la subjetividad introducida por el agricultor pueden ocasionar fallas en el diagnostico.

Por esta razón, se implementó un sistema capaz de detectar la presencia del gorgojo de los Andes. Para esto se emplea visión artificial para realizar el preprocesamiento de imágenes extraídas de fotografias proporcionadas por agricultores.

Además, se desarrolla un modelo de aprendizaje profundo basado en la arquitectura VGGNet. La arquitectura fue llevada a una aplicación móvil mediante la herramienta el modelo denominado MobileNet. Los resultados arrojaron, un índice de reconocimiento adecuado, obteniendo una precisión en la predicción hasta del $84 \%$.

Palabras claves -- Aprendizaje profundo, red neuronal, foliolo, preprocesamiento, aplicación móvil

Abstract - Various methods are employed to prevent potato crops from being affected by diseases and pests, one of which is monitoring, which consists of people walking through the crops and using their cognitive abilities to recognize the presence of pests. However, limitations in human capacity such as inaccuracy due to the subjectivity introduced by the farmer can cause failures in the diagnosis.

For this reason, a system capable of detecting the presence of the Andean weevil was implemented. For this purpose, artificial vision is used to perform the preprocessing of images extracted from photographs provided by farmers.

In addition, a deep learning model based on the VGGNet architecture was developed. The architecture was taken to a mobile application using the model called MobileNet. The results showed an adequate recognition rate, obtaining a prediction accuracy of up to $84 \%$.

Keywords -- Deep learning, neural network, foliolus, preprocessing, mobile application.

Digital Object Identifier (DOI):

http://dx.doi.org/10.18687/LEIRD2021.1.1.42

ISSN: 2414-6390 ISBN: 978-958-52071-9-6

$1^{\text {sh }}$ LACCEI International Multiconference on Entrepreneurship, Innovation and Regional Development - LEIRD 2021: "Ideas to Overcome and Emerge from the Pandemic Crisis", Virtual Edition, December 9-10, 2021. 


\section{Co-diseño de una aplicación para el reconocimiento in situ del gorgojo de los andes en cultivos de papa}

\begin{abstract}
Resumen -Diversos métodos son empleados evitar que los cultivos de papa se vean afectados por enfermedades y plagas, uno de estos, es el monitoreo que consiste en personas encargadas de recorrer los cultivos y emplear sus capacidades cognitivas para reconocer la presencia de plagas. Sin embargo, limitaciones en la capacidad humana como la imprecisión por la subjetividad introducida por el agricultor pueden ocasionar fallas en el diagnostico.

Por esta razón, se implementó un sistema capaz de detectar la presencia del gorgojo de los Andes. Para esto se emplea visión artificial para realizar el preprocesamiento de imágenes extraídas de fotografias proporcionadas por agricultores.

Además, se desarrolla un modelo de aprendizaje profundo basado en la arquitectura VGGNet. La arquitectura fue llevada a una aplicación móvil mediante la herramienta el modelo denominado MobileNet. Los resultados arrojaron, un índice de reconocimiento adecuado, obteniendo una precisión en la predicción hasta del $84 \%$.
\end{abstract}

Palabras claves -- Aprendizaje profundo, red neuronal, foliolo, preprocesamiento, aplicación móvil

Abstract - Various methods are employed to prevent potato crops from being affected by diseases and pests, one of which is monitoring, which consists of people walking through the crops and using their cognitive abilities to recognize the presence of pests. However, limitations in human capacity such as inaccuracy due to the subjectivity introduced by the farmer can cause failures in the diagnosis.

For this reason, a system capable of detecting the presence of the Andean weevil was implemented. For this purpose, artificial vision is used to perform the preprocessing of images extracted from photographs provided by farmers.

In addition, a deep learning model based on the VGGNet architecture was developed. The architecture was taken to a mobile application using the model called MobileNet. The results showed an adequate recognition rate, obtaining a prediction accuracy of up to $84 \%$.

Keywords -- Deep learning, neural network, foliolus, preprocessing, mobile application.

\section{INTRODUCCIÓN}

La agricultura desempeña un papel fundamental para la economía mundial debido a que no solo representa más del $27 \%$ del PIB en algunos países y genera según la Agencia de Protección Ambiental unos 330.000 millones de dólares de beneficios anuales en todo el mundo, sino que, además, es particularmente eficaz para reducir el hambre y la malnutrición [1]. En el transcurso del tiempo, este sector ha sufrido varios cambios para satisfacer el aumento de la población mundial que se ha duplicado en los últimos 50 años, [2] esto sumado al aumento de personas que migran desde zonas rurales a zonas urbanas, ha ocasionado que la productividad agrícola tenga que aumentar de forma sostenible y más independiente del trabajo humano.

Desde la década de 1990 se realizaron varios estudios para mejorar la eficiencia de la producción, originando el concepto de "agricultura de precisión" (PA - Precision Agriculture, por sus siglas en inglés), una noción de gestión agrícola basada en la observación, evaluación y control ante la variabilidad de los cultivos, con el objetivo de optimizar los rendimientos preservando los recursos [3]. La agricultura de precisión permite a los agricultores tomar decisiones en aspectos como: la selección de semillas, el seguimiento de enfermedades, la identificación de malas hierbas y el uso de pesticidas y fertilizantes.

Recientemente, las tecnologías existentes en la industria como la teledetección, el Internet de las Cosas (IoT - Internet of Things, por sus siglas en inglés) y las plataformas robóticas se han incorporado en la agricultura, dando lugar al concepto de "agricultura inteligente". La agricultura inteligente ha permitido afrontar retos de la producción agrícola en términos de impacto medioambiental, seguridad alimentaria y sostenibilidad [4], manteniendo la disponibilidad y la alta calidad nutricional de los productos en todo el planeta mediante el uso de procedimientos agrícolas sostenibles.

Para hacer frente a los retos complejos e imprevisibles de los ecosistemas agrícolas, es necesario realizar un seguimiento y comprensión de diversas variables y fenómenos físicos; esto implica, la creación de grandes volúmenes de datos y del uso de las nuevas tecnologías de la información y las comunicaciones, tanto para la gestión de cultivos como para la observación de ecosistemas [5]. La recolección de información permite la construcción de bases de datos, que serán utilizadas en el desarrollo de sistemas para la detección de plagas, enfermedades, entre otros. Para ello las imágenes deben ser procesadas mediante técnicas basadas en procesos de aprendizaje automático como k-means, máquinas de vectores de soporte (SVM - Support Vector Machine, por sus siglas en inglés) y redes neuronales artificiales (RNA).

El aprendizaje automático (ML - Machine Learning, por sus siglas en inglés) ha surgido junto con tecnologías como el 
Big data y la informática de alto rendimiento para crear nuevas oportunidades de cuantificar y comprender los procesos con gran cantidad de datos en entornos operativos agrícolas. El ML se define como el campo científico que otorga a las máquinas la capacidad de aprender sin estar estrictamente programadas [6].

A partir del constante desarrollo y de los aportes significativos en el campo de la inteligencia artificial, surge una rama del aprendizaje automático llamado aprendizaje profundo, conocido más comúnmente como Deep Learning (DL) que permite que modelos computacionales compuestos por múltiples capas de procesamiento aprendan representaciones de datos con múltiples niveles de abstracción [7]; esta técnica permite desarrollar aplicaciones para el procesamiento de imágenes, video, voz y audio, así como el reconocimiento visual de objetos o datos secuenciales como texto y habla.

Con el entusiasmo masivo que se ha puesto en el campo del aprendizaje profundo se han conseguido grandes mejoras en los últimos años. El aprendizaje profundo ha llamado la atención de la agricultura, donde se han desarrollado soluciones enfocadas al reconocimiento de imágenes para la detección de enfermedades de las plantas, el control de las malas hierbas y el recuento de las plantas. Además, el aprendizaje profundo ha permitido numerosos avances en cuanto al control de plagas evitando el uso ciego de plaguicidas que da lugar a cultivos insalubres y daños medioambientales, pues anteriormente la detección de plagas se llevaba a cabo mediante la búsqueda y observación por parte del agricultor, lo cual es evidentemente laborioso y propenso a errores [8].

El presente proyecto de investigación desarrollado por el grupo de investigación en procesamiento de señales DSPUPTC, tiene por objeto el desarrollo de una aplicación en Android, con el fin de emplear la tecnología con la que cuentan los agricultores como herramienta para identificar la presencia de la plaga in situ empleando métodos de aprendizaje profundo. El proyecto plantea el desarrollo de un sistema de inteligencia artificial basado en aprendizaje profundo como método de procesamiento de imágenes para la detección de la presencia del gorgojo de los Andes y la clasificación de plantas a partir del reconocimiento de patrones visuales presentes en las hojas. Este trabajo se articula con las líneas de procesamiento digital de señales y tecnología agropecuaria y para el medio ambiente, dada la naturaleza de la investigación y los productos generados.

\section{MetodologíA}

La propuesta se enmarca en una investigación aplicada, de metodología experimental debido a que se pondrán a prueba elementos teóricos del procesamiento de imágenes por medio de un proceso participativo con la comunidad para generar una alternativa conjunta, con enfoque mixto, desde una mirada cualitativa dirigida a la construcción por medio de procesos de creatividad colectiva con los expertos de las realidades estudiadas; las experiencias basadas en el codiseño serán transversales en el desarrollo del proyecto ya que de acuerdo con quienes proponen un marco para generar propuestas de codiseño basadas en relaciones dinámicas, el desarrollo del proyecto implementa etapas basadas en i) un enfoque abierto, ii) promover el propósito de cambio, iii) creación conjunta, iv) diversidad del conocimiento, v) integración de actores y vi) diversidad en las experiencias creativas. Desde la mirada cuantitativa, dada la naturaleza de los datos obtenidos y el proceso para realizarlos, se llevan a cabo por medio del diseño de un algoritmo que brinde eficiencia en el reconocimiento de parámetros para el procesamiento digital de las imágenes.

El presente trabajo aborda tres fases metodológicas: exploración, dirigida a determinar las características de las imágenes y reconocimiento del entorno en el cual se diseñará la aplicación, así como las especificaciones de los dispositivos móviles que utilizan los agricultores para tomar las fotografías.; la segunda fase desarrollo, se centrará en el diseño y elaboración del algoritmo y su aplicación en el procesamiento de las imágenes en conjunto con métodos de inteligencia artificial, así como, la creación de la aplicación móvil. Finalmente, la fase de evaluación, con el fin de testear el correcto funcionamiento de la aplicación y evaluar su desempeño a partir de métricas.

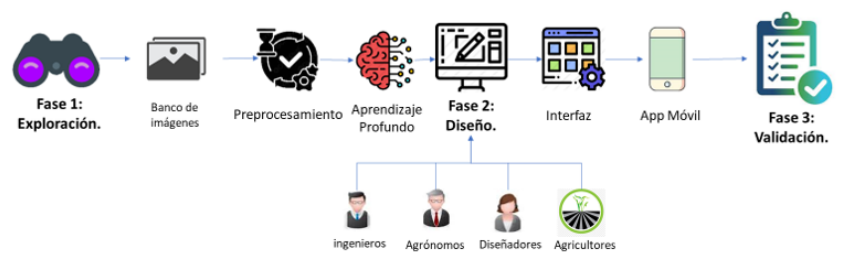

Figura 1. Diagrama de desarrollo metodologico.

\section{A. Conformación del conjunto de datos.}

Las imágenes de foliolos de plantas de cultivos de papa, utilizadas para la conformación del conjunto de datos del presente trabajo, fueron extraídas manualmente por los autores de fotografías tomadas por la comunidad cultivadora de papa, quienes participaron en una campaña para el desarrollo colaborativo del proyecto de investigacion. Las fotografías corresponden a sembríos ubicados en Duitama, Tibasosa, Sogamoso, Tunja, Aquitania, Ventaquemada, Monguí y Corrales (Boyacá, Colombia), estas fueron capturadas por diversas personas en diferentes días, por lo cual, poseen condiciones de iluminación y enfoque (resolución, nitidez y contraste) variados, así como fotografías con dimensiones que van desde 1280x960 hasta 4160x2336. 


\section{B. Preprocesamiento de imágenes.}

El principal reto en términos de análisis de imágenes es lograr una discriminación adecuada entre un folíolo y el fondo de la fotografía, que puede ser maleza, suelo u otros folíolos; de modo que el algoritmo sea capaz de entrenarse y clasificar imágenes de manera adecuada.

\section{Diseño de la red neuronal convolucional.}

La red toma como entrada una imagen y la trata como una matriz de dimensiones número de píxeles $\mathrm{x}$ número de píxeles $\mathrm{x}$ número de canales de color. Para realizar la convolución se necesita un filtro, en ingles kernel, que se encargará de extraer las características de la imagen en cada capa, este proceso podría interpretarse como transformaciones abstractas de la imagen. La convolución consiste en desplazar el kernel por cada uno de los elementos de la imagen original e ir operando matemáticamente (producto escalar) la matriz y el filtro obteniendo así el mapa de características. De esta forma, el núcleo recorre todas las neuronas de entrada y genera una nueva matriz, que será la entrada de una nueva capa de neuronas oculta.

La capa convolucional tiene como objetivo aprender representaciones de características de las entradas, la capa convolucional se compone de varios núcleos de convolución que se utilizan para calcular diferentes mapas de características.

\section{Desarrollo de la aplicación móvil}

La aplicación permite por medio de la cámara del dispositivo móvil, la captura y clasificación de fotografías de folíolos de papa, mostrando como resultado la probabilidad entre sí presenta o no afectación por el gorgojo de los Andes. Debido a que el público objetivo posiblemente son usuarios inexpertos en el manejo de tecnología, la aplicación es de fácil instalación, requiere solo un permiso para el acceso a la cámara el cual, solo es solicitado la primera vez que se ingresa, cuenta con una interfaz intuitiva compuesta por dos botones, uno para iniciar la detección y el otro para el cambio de cámara.

\section{DESARROLLO}

Inicialmente se utilizó un conjunto de datos de 1530 imágenes las cuales fueron clasificadas, con la ayuda de ingenieros agrónomos, en dos clases, folíolos afectados y folíolos sanos; teniendo 765 imágenes correspondientes a folíolos sanos y la cantidad restante correspondientes a folíolos afectados por el gorgojo de los Andes. En los entrenamientos iniciales no se obtuvieron resultados satisfactorios a pesar de realizar ajustes en los hiperparámetros, razón por la cual se decidió emplear el aumento de datos a través de operaciones de rotación y escalado.

El criterio empleado para definir el conjunto de datos de entrenamiento y el conjunto de datos de prueba es el siguiente: dos tercios de los datos fueron empleados para el entrenamiento y el tercio restante fue utilizado para pruebas.

Las imágenes fueron sometidas a un procesamiento offline, en un entorno gráfico de desarrollo para Python ${ }^{\mathrm{TM}}$, utilizando la librería OpenCV. El sistema de procesamiento de imágenes distingue cuatro componentes que se le aplicaran secuencialmente a la imagen de entrada antes de que llegue al modelo de aprendizaje profundo: a) suavizado de la imagen, b) detección del color verde, c) segmentación con umbralización, y d) operación entre bits de conjunción.

Suavizado de la imagen: esta etapa fue implementada usando una función para la eliminación de ruido de imágenes en color que hace parte de la librería OpenCV denominada fastNlMeansDenoising Colored.

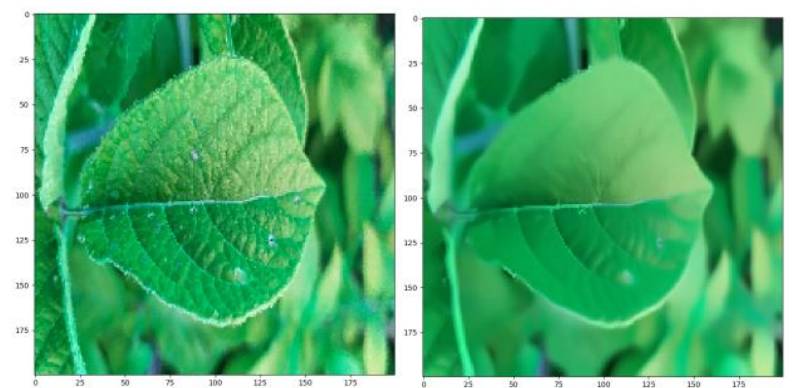

Figura 2. Imagen de un folíolo sano. A la izquierda se presenta la imagen original mientras que a la derecha se presenta la imagen después de realizar un suavizado.

$>$ Detección del color verde en el espacio de color HSV: el objetivo del segundo paso es detectar y filtrar el rango que representa el color verde en el espacio de color HSV, definiendo previamente los umbrales máximos y mínimos. Este modelo de color fue seleccionado debido a la robustez que presenta en tareas de separación de color.
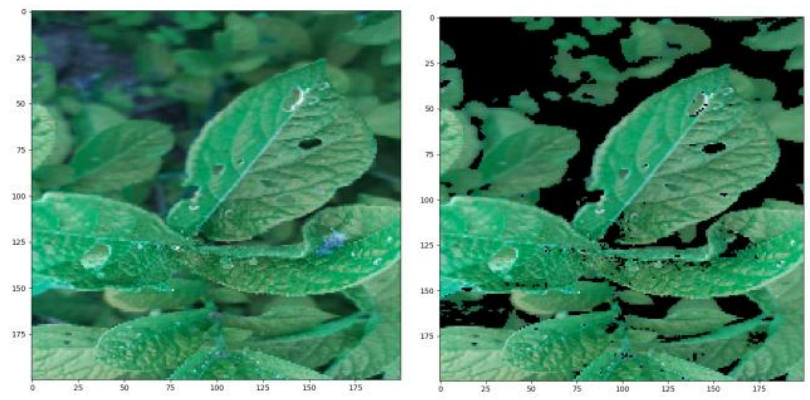

Figura 3. Imagen de un folíolo afectado. A la izquierda se encuentra la imagen original, a la derecha se presenta la 
imagen después de realizar la operación de umbralización del color verde en el espacio HSV.

Segmentación del color con umbralización: esta técnica permite identificar mejor las hojas de la planta que se encuentran próximas a la cámara y en las cuales el reconocimiento de la plaga tendrá un porcentaje menor de error. Para esta etapa la imagen leída en formato RBG es llevada a escala de grises. Posteriormente se realiza umbralización con el método de Otsu Posteriormente, la imagen segmentada pasa por un filtro morfológico de cierre que brinda la posibilidad de eliminar pequeños agujeros y rellenar brechas en los contornos que en caso de no corregirlos se pueden convertir en ruido para el algoritmo por lo que podría ocasionar errores en los resultados.
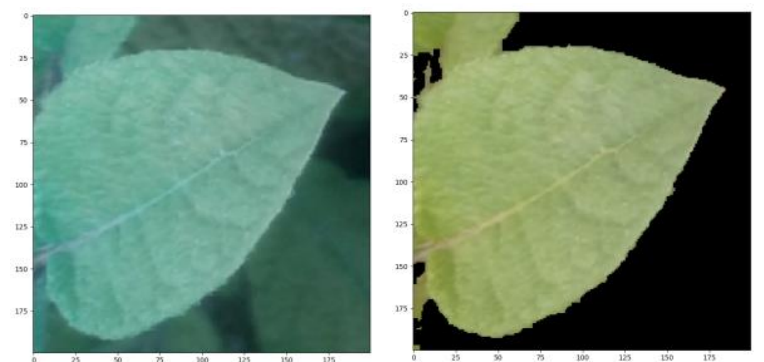

Figura 4. Imagen de un folíolo sano. A la izquierda se encuentra la imagen original, a la derecha se presenta la imagen después de realizar la operación de segmentación con umbralización.

$>$ Operación entre bits de conjunción: en esta etapa se implementó una operación de conjunción (AND) entre los elementos de las matrices de correspondientes a las imágenes suavizada, umbralizada y con detección de verde, obteniendo una imagen donde se separa el fondo, de las hojas de la planta y se descarta posibles objetos que no sean los folíolos de la planta de papa a analizar
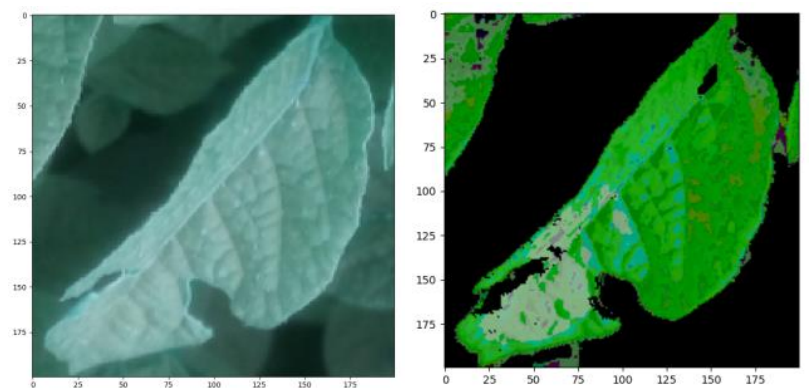

Figura 5. Imagen de un folíolo afectado. A la izquierda se encuentra la imagen original, a la derecha se presenta la imagen después de realizar la operación de conjunción entre las imágenes suavizada, umbralizada y segmentada.
Sin embargo, a pesar de emplear estas técnicas por separado y de forma individual, resulta complejo configurar los parámetros establecidos en estas operaciones de modo que funcionen para una gran variedad de imágenes pertenecientes a foliolos, donde predomina la diferencia de tonalidad en el color de las hojas, los sombras sobre foliolos que el algoritmo puede interpretar erróneamente suprimiendo regiones que no debería y otras características que hacen que foliolos sanos puedan llegar al modelo de aprendizaje profundo como foliolos afectados y en consecuencia, generar predicciones erradas. Por esta razón, las imágenes que componen el conjunto de datos con el que se entrena el modelo de inteligencia artificial son fotografías a las que solo se les hizo escalado de forma que todas tuvieran la misma forma, un cuadrado pequeño de $200 \times 200$.
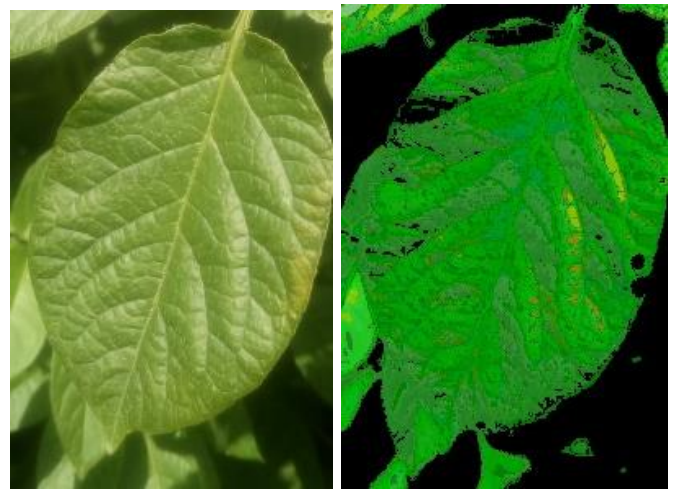

Figura 6. Errores introducidos al realizar el preprocesamiento imágenes de folíolos sanos.

Con respecto al diseño de la red de aprendizaje profundo basada en la arquitectura VGG, está compuesta por bloques cada uno como una combinación de una capa convolucional con una de agrupamiento (pooling). La estructura VGG para el diseño de la red multicapa fue escogido por su alto desempeño en tareas de clasificación y detección de objetos en imágenes encontrados en la literatura. Los hiperparámetros ajustados fueron: la cantidad de bloques, el número de filtros de cada bloque, el tamaño del lote, el número de iteraciones, el tamaño del conjunto de datos, el optimizador y el enfoque para mejorar la tasa de aprendizaje.

Para el desarrollo de la aplicación móvil se emplea la herramienta "Personal Image Classifier". En primer lugar, se establecen las etiquetas de "Afectadas" y "No Afectadas". Luego, se realiza la configuración del modelo basado en las pruebas realizadas en el algoritmo desarrollado. Los parámetros a configurar en este paso son: tamaño del núcleo de convolución, tasa de aprendizaje, iteraciones o épocas y el optimizador, adicionalmente se establecerá la secuencia definida por la arquitectura VGG.

Una vez configuradas las características y parámetros, se entrena el modelo Al terminar el entrenamiento del modelo se 
cargan las imágenes correspondientes al conjunto de datos de validación para visualizar el rendimiento del modelo.

La aplicación móvil es desarrollada con MIT App Inventor, la interfaz se divide en tres secciones, en la primera se podrá visualizar lo captado por la cámara del dispositivo, en la segunda se podrá ver el nivel de confianza de la detección visualizados y por último se tiene los botones de funcionamiento, los cuales permiten girar la cámara y realizar la detección.
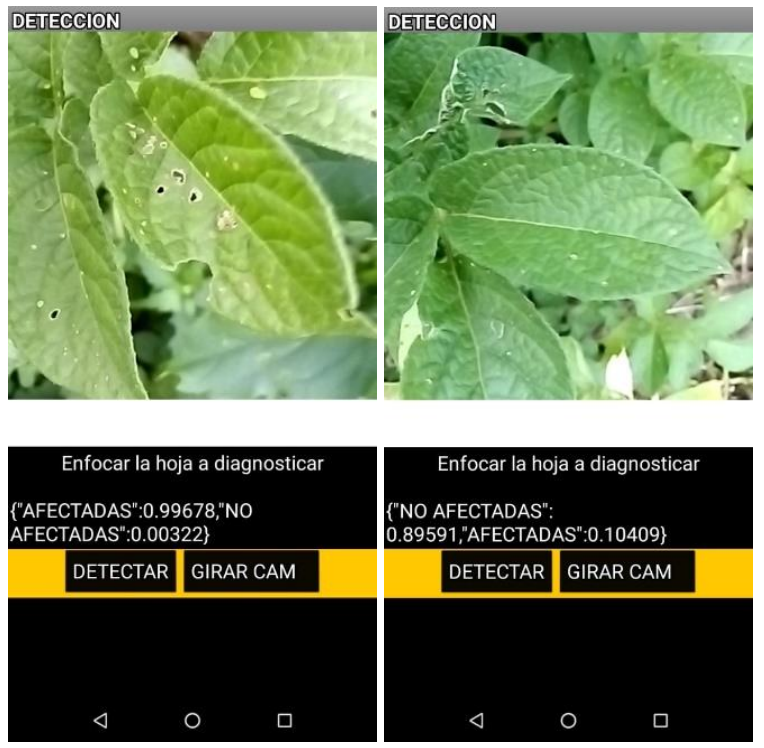

Figura 7. Resultados de la aplicación con dispositivo móvil.

\section{ANÁLISIS DE RESULTADOS}

El rendimiento de la red neuronal convolucional propuesta se evaluó con 460 imágenes pertenecientes tanto a foliolos afectados como no afectados disponibles en el conjunto de datos que se describe en la sección II, aparta A (Conformación del conjunto de datos). Se han utilizado cuatro algoritmos de aprendizaje profundo diferentes. Todos los algoritmos ofrecen diferentes tipos de rendimiento en función de los diferentes hiperparámetros.

Se han realizado pruebas con redes neuronales convolucionales basadas en arquitectura VGG de uno, dos y tres bloques. Para la red neuronal convolucional se han utilizado tres bloques con optimizador ADAM y una técnica de aumento de datos artificial aleatoria a partir de rotaciones y escalado.

Tabla 1. Tabla de precisión y porcentaje de perdidas.

\begin{tabular}{|c|c|c|}
\hline Método & $\begin{array}{c}\text { Porcentaje de } \\
\text { precisión de datos } \\
\text { de validación }\end{array}$ & $\begin{array}{c}\text { Porcentaje de } \\
\text { pérdidas de entropía } \\
\text { cruzada con datos de } \\
\text { validación }\end{array}$ \\
\hline
\end{tabular}

\begin{tabular}{|l|l|l|}
\hline $\begin{array}{l}\text { Red neuronal convolucional } \\
\text { de } 1 \text { bloque }\end{array}$ & $66.66 \%$ & $60.14 \%$ \\
\hline $\begin{array}{l}\text { Red neuronal convolucional } \\
\text { de } 2 \text { bloques }\end{array}$ & $72.00 \%$ & $58.01 \%$ \\
\hline $\begin{array}{l}\text { Red neuronal convolucional } \\
\text { de } 3 \text { bloques }\end{array}$ & $74.86 \%$ & $49.87 \%$ \\
\hline $\begin{array}{l}\text { Red neuronal convolucional } \\
\text { de } 3 \text { bloques con aumento de } \\
\text { datos artificial. }\end{array}$ & $85.72 \%$ & $39.54 \%$ \\
\hline
\end{tabular}

De la tabla 1, se observa que la implementación de red neuronal convolucional que proporciona la mayor precisión $(85.75 \%)$ es la red VGG de 3 bloques con aumento de datos artificial, seguida de la red neuronal convolucional de 3 bloques. Cuando se implementa un modelo de este tipo se observa que al 10 iteraciones o épocas se tiende a tener sobreajuste (overfitting en inglés) y que al aumentar el número de capas se aumenta el porcentaje de precisión en la clasificación, sin embargo, aumenta la complejidad de la red y con ello el tiempo requerido para los entrenamientos.

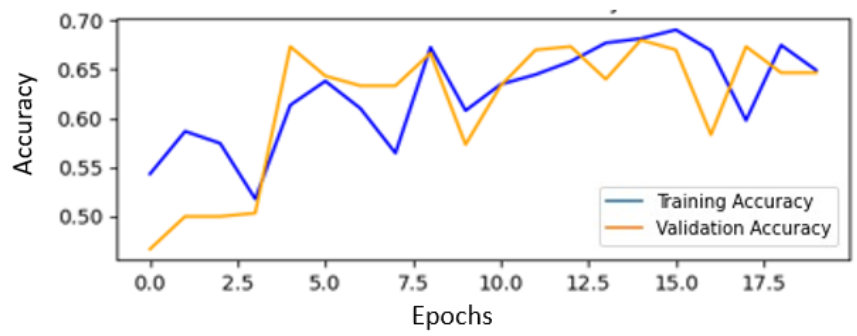

Figura 8. Gráfica de precisión para modelo VGG de un bloque.

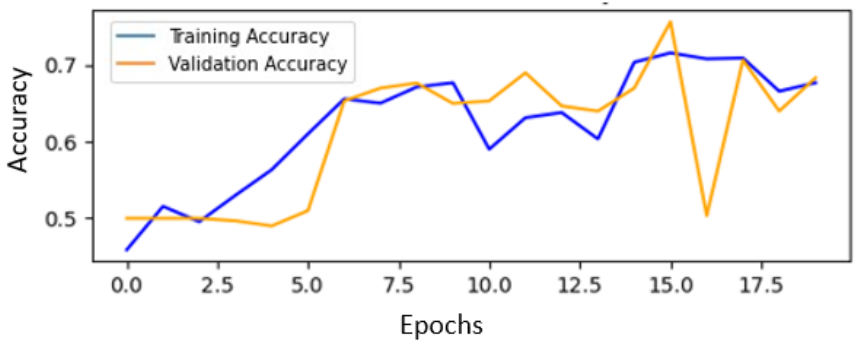

Figura 9. Gráfica de precisión para modelo VGG de dos bloques.

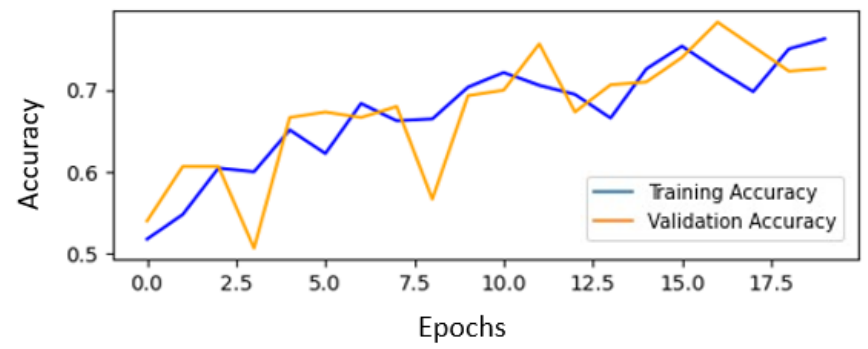

Figura 10. Gráfica de precisión para modelo VGG de tres bloques. 

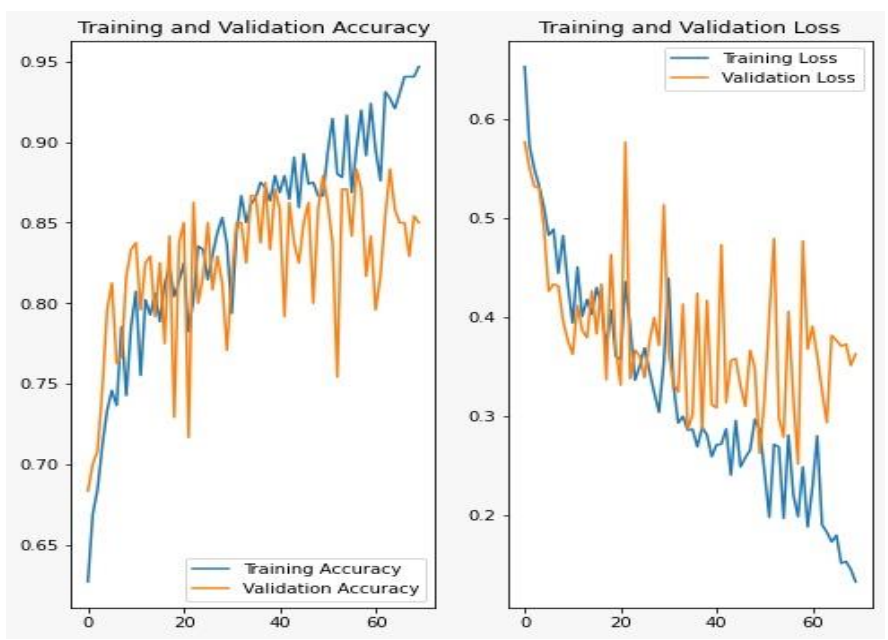

Figura 11. Gráfica de precisión para modelo VGG de tres bloques con aumento de datos artificial.

\section{CONCLUSIONES}

El presente trabajo se enfocó al análisis de fotografías de foliolos pertenecientes a cultivos de papa con el fin de detectar el daño ocasionado por el gorgojo de los Andes, para lo cual, se compararon redes neuronales de varias capas basados en la arquitectura VGG (Visual Geometry Group) modificando sus hiperparámetros. En el sistema de una sola copa se encontró un porcentaje de error de alrededor de $60 \%$, este error es ocasionado por la estructura de la red, dada su baja profundidad y una base de datos limitada que solo consideraba, inicialmente, 1530 imágenes. Para el caso del sistema de tres capas de aprendizaje profundo se obtuvo un porcentaje de error en el reconocimiento de la afectación del gorgojo menor del $40 \%$, en el conjunto de imágenes utilizadas para la validación, y menos del $20 \%$ en el conjunto de entrenamiento.

Al intentar construir las redes neuronales convolucionales basadas en VGG, se observa que no existen reglas generales para mejorar los resultados, sino que es necesario un estudio heurístico. Además, se resalta la importancia de establecer un tratamiento adecuado para el conjunto de imágenes con el que se entrena el algoritmo, debido a que la capacidad que tendrá el modelo para lograr la abstracción depende de las características de cada imagen. Dado que las propiedades de las fotografías, como condiciones de iluminación, no se encuentran estandarizadas pues provienen de diversos usuarios, resulta complejo establecer un preprocesamiento de las imágenes adecuado ante la variabilidad y el estado de crecimiento continuo de los cultivos. Debido a lo anterior, como un aspecto importante dentro del procesamiento de imágenes del cultivo de la papa, se propone el aumento de la base de datos para así tener la posibilidad de entrenar modelos más robustos.
El sistema de redes neuronales fue validado en campo mediante el desarrollo de una aplicación móvil construida en el entorno App Inventor, la cual permite generar un modelo de clasificación basado en MobilNet donde se replicó la red VGG de tres capas. Las pruebas se realizaron empleando cuatro dispositivos móviles con diferentes especificaciones técnicas, dentro de las cuales destaca la resolución de la cámara como una característica determinante en la respuesta del modelo debido a que celulares con bajas resoluciones, es decir, una resolución similar a la empleada en la construcción de la base de datos, tenían un porcentaje de efectividad más alto en comparación a celulares con mejor definición de imagen.

De la experiencia desarrollada se destaca una vez más la creciente importancia de los sistemas de detección y clasificación de imágenes basados en redes neuronales convolucionales como una herramienta que permita confirmar o descartar la presencia de la plaga, sin que esto signifique el reemplazar el criterio basado en la experiencia de los agricultores ni los conocimientos de especialistas en estas áreas.

\section{REFERENCIAS}

[1] NACIONES UNIDAS PARA LA AGRICULTURA Y LA ALIMENTACIÓN. La contribución del crecimiento agrícola a la reducción de la pobreza, el hambre y la malnutrición. El estado de seguridad alimentaria en el mundo. (2012). [Consultado: 28 de julio de 2021]. Disponible en: http://www.fao.org/3/i3027s/i3027s04.pdf

[2] Kitzes, J., Wackernagel, M., Loh, J., Peller, A., Goldfinger, S., Cheng, D., Tea, K., 2008. Shrink and share: humanity's present and future Ecological Footprint. Philosophical Transactions of the Royal Society B 363, 467475.

[3] McBratney, A., Whelan, B., Ancev, T. et al. Future Directions of Precision Agriculture. Precision Agric 6, 7-23 (2005). https://doi.org/10.1007/s11119-005-0681-8

[4] Gebbers, Robin \& Adamchuk, Viacheslav. (2010). Precision Agriculture and Food Security. Science327(5967), 828-831. Science (New York, N.Y.). 327. 828-31. 10.1126/science.1183899.

[5] A Kamilaris, A Kartakoullis, FX Prenafeta-Boldú. Computers and Electronics in Agriculture 143, 23-37, 2017. 398, 2017.

[6] De Oliveira, L. T., Thomaz, C. D. A., de Carvalho, L. M. T., Lacerda, W. S., Samuel, R., \& Martinhago, A. Z. (2007). Comparison of Machine Learning Algorithms for Mapping the Phytophysiognomies of the Brazilian Cerrado. In GeoInfo (pp. 195-205).

[7] LeCun, Y., Bengio, Y., \& Hinton, G. (2015). Deep learning. nature, 521(7553), 436-444.

[8] Liu, L., Xie, C., Wang, R., Yang, P., Sudirman, S., Zhang, J., ... \& Wang, F. (2020). Deep learning based automatic multi-class wild pest monitoring approach using hybrid global and local activated features. IEEE Transactions on Industrial Informatics. 